\title{
FLOW SHOP SCHEDULING USING CLUSTERED DIFFERENTIAL EVOLUTION
}

\author{
Donald Davendra and Ivan Zelinka, \\ Department of Applied Informatics, \\ Tomas Bata University in Zlin, \\ Nad Stranemi 4511, Zlin 76001 \\ Czech Republic. \\ Email: \{davendra,zelinka\}@fai.utb.cz
}

\section{KEYWORDS}

Differential evolution, flowshop scheduling, clustering

\begin{abstract}
A generic approach of population dynamics and clustering for permutative problems is presented in this paper. Diversity indicators are created from solution ordering and its mapping is shown as an advantage for population control in metaheuristics. Differential Evolution Algorithm is embedded with a population based on this approach and vetted with the Flow shop scheduling problem. Extensive experimentation is conducted on benchmark problems in this area with good results.
\end{abstract}

\section{INTRODUCTION}

Metaheuristics are algorithms, which are used for the optimization of complex systems. The vital attribute for these heuristics is that they have to operate without apriori information of the system.

Metaheuristics operate on two ideological frameworks, firstly that a group of solutions provide a better platform to find optimal solution, and secondly that certain guiding concept leads the solutions towards the optimal solution, or nearby regions.

A number of different transformation concepts have evolved within the scope of Metaheuristics (Dreo et al., 2006). Genetic Algorithms, Differential Evolution, Particle Swarm Optimization and Self Organising Migrating Algorithm are some of the most potent heuristics available. Most of these algorithms have mimicked naturally occurring phenomena.

The principle concept of the population is to provide a pool of solutions from which the optimal solution evolves from, however during the subsequent transformation, the solutions cluster together in nearby neighbourhoods. This leads to the loss of diversity of the solutions, and later generations are usually mutating within a cloned gene pool. This phenomenon is generally termed as stagnation and the two most current correcting tools are local search within the hierarchical population and forfeiting of some better-placed solutions in lieu of worst ones (Onwubolu (2002)).

\author{
Godfrey Onwubolu, \\ Knowledge Management \& Mining, \\ Richmond Hill, \\ Canada. \\ Email: onwubolu_g@dsgm.ca
}

Stagnation is a major concern for evolutionary heuristics, since evolution is principally based on diversity of the existing population. The preconception that extended generations of a heuristic will lead to better solutions is nonviable for a stagnated population.

This paper is devoted to the concept of the population and its controlling dynamics. It is shown through experiment that population control is an effective tool in sustaining the diversity of the population, which in turn leads to more viable regions of search space for exploration.

\section{POPULATION DYNAMICS}

One of the most challenging optimization problems is permutative based combinatorial optimization. This class of problem, harbours some of the most famous optimization problems like travelling salesman (TSP) and vehicle routing problem (VRP).

What makes a permutative problem complex is that the solution representation is very concise, since it must have a discrete number of values, and each occupied variable in the solution is unique. Given a problem of size $n$, a representation can be described as $x=\left\{x_{1}, x_{2}, x_{3}, \ldots, x_{n}\right\}$, where each value $x_{1}$ in the solution is unique and the entire set of solutions is an integer representation from $\{1$, $n\}$.

From an optimization point of view, this represents a number of problems. Firstly, the search space is discrete and a number of validations inevitably have to be conducted in order to have a viable solution. Secondly, the search space is very large, to the scale of $n$ !. Consequently, these problems are generally termed $N P$ or $N P$ Hard (Hochbam (1997)).

The usual approach is to explore the search space in the neighbourhood of good solutions in order to find better solutions. This unfortunately has the effect of converging the population, which then leads to stagnation. The usual term is local optima convergence/stagnation.

This research looks at the diversity of the population in order to aid the application of metaheuristics. A permutative solution and its representation present some advantages to this effect. The usual measure of a solution is its fitness, in respect to the problem being solved. In a permutative solution, the distinct ordering of values gives 
the opportunity to have other measures of diversity.

The following sections describe the approach developed within this scope of research. The first part discusses the need for having distinct initial population. The second part describes some fields of measure of diversity followed by the approach of clustering of the solutions. Differential Evolution Algorithm is then presented to which the clustered population has been applied. The subsequent sections describe the extensive experimentation conducted on Quadratic Assignment problems and analysis of the dynamic nature of clustering populations.

\subsection{Initial Population}

The basic concept is to have a "clustured" population. This is an ideal position from which to guage the effectiveness of the heuristic. Also, this provides a better initial point for scheduling problems. An ideal explanation is the TSP, where adjacent cities usually provide minimal travel distance.

The initial population, $P$, for this heuristic is partially stochastic and partly deterministic. The population is divided into $n$ sub-populations, $S P S, \mathrm{n} / 2$ randomly generated $\left(S P_{\text {rand }}\right)$ and the rest structurally generated (SP $\left.P_{\text {struct }}\right)$.

The formulation for $S P_{\text {rand }}$ is fairly simple. A random permutative string is generated for each solution till a specified number given as $P_{\text {size }}$.

The structured population $S P_{\text {struct }}$ is somewhat more complex. It is made of two parts. In the first part an initial solution is generated with ascending values given as $x_{\text {ascending }}=\{1,2,3, . ., n\}$, where $n$ is the size of the problem. In order to obtain a structured solution, the first solution is segmented and recombined in different orders to produce different combinations. The first segmentation occurs at $n / 2$ and the two halvesś are swapped to produce the second solution. The second fragmentation occurs by the factor $3 ; n / 2$. Three regions of solutions now exist. The number of possible recombination's that can exist is $3 !=6$. At this point there are nine solutions in the $S P_{\text {struct }}$. The general representation is given as:

$$
k \geq 1+2 !+3 !+\ldots .+z !
$$

where $z$ is the total number of permutations possible and $k$ is $P_{\text {size }} / n$.

The second part of $S P_{\text {struct }}$ is made from solution in descending order $x_{\text {desending }}=\{n, \ldots ., 2,1\}$. A similar approach is used for generating the rest of the possible permutations. The end result is that $n$ separate populations exist and are used independently by the underlying heuristic.

\subsection{Solution Dynamics}

A solution represented as $x=\left\{x_{1}, x_{2}, \ldots, x_{n}\right\}$, where $n$ is the number of variables, within a population has a number of attributes. Usually the most visible is its fitness value, by which it is measured within the population. This approach is not so viable in order to measure
Table 1: Solution Parameters

\begin{tabular}{|l|l|l|}
\hline Parameter & Description & Activity \\
\hline Deviation & Measure of deviation & Control \\
\hline Spread & Alignment of solution & Control \\
\hline Life & Generation cycles & Selection \\
\hline Offspring & No. of offspring's & Selection \\
\hline
\end{tabular}

Table 2: Spread generalization

\begin{tabular}{|c|c|}
\hline Spread & Generalization \\
\hline$>0$ & Forward spread \\
\hline 0 & Even spread \\
\hline$<0$ & Reverse spread \\
\hline
\end{tabular}

the diversity of the solution in the population. In retrospect, a single solution is assigned a number of attributes for measure, as given in Table 1. The most important attribute is the deviation (the difference between successive values in a solution). Since we are using only permutative solutions, deviation or ordering of the solution is important. This is due to the fact that each value in the solution is unique. Each value in the solution has a unique footprint in the search space. The formulation for deviation is given as:

$$
\delta=\left(\frac{\sum_{i=1}^{n-1}\left|x_{i}-x_{i+1}\right|}{n}\right) x_{i} \in\left\{x_{1}, x_{2}, \ldots, x_{n}\right\}
$$

Spread of a solution gives the alignment of the solution. Each permutative solution has a specific ordering, whether it is forward aligned or reverse aligned. Whereas deviation measures the distance between adjacent solutions, spread is the measure of the hierarchy of subsequent solutions given as:

$$
\partial=\left\{\begin{array}{c}
+1 \quad \text { if }\left(x_{i+1}-x_{i}\right) \geq 1 \\
-1 \quad \text { if }\left(x_{i+1}-x_{i}\right) \leq 1 \\
i \in\{1,2, \ldots, n\}
\end{array}\right.
$$

The generalisation of spread is given in Table 2. Life is the number of generations the solution has survived in the population and Offspring is the number of viable solutions that have been created from that particular solution. These two variables are used for evaluating the competitiveness of different solutions.

\subsection{Clustering}

Within the population, certain solutions exhibit attracting features. These points are usually local optima regions, which draw the solutions together. The approach utilized is to subdivide the population in clusters, each cluster a distinct distance from another. Two controlling parameters are now defined which control the clusters.

Cluster Attractor $C_{A}$ : The distance that each segment of solution has to differ from each other. The $C_{A}$ is given in (4).

$$
C_{A} \in[0.1,1+)
$$


Table 3: Illustrative example

\begin{tabular}{|l|c|c|c|c|c|}
\hline Solution & Dev & Spread & Clust & $C_{A}$ & Fitness \\
\hline 12345678910 & 0.9 & +9 & 1 & 1.2 & 1592 \\
\hline 12569103478 & 2.1 & +7 & 2 & 1.1 & 1559 \\
\hline 10987654321 & 0.9 & -9 & 1 & 1.2 & 1567 \\
\hline 10965218743 & 2.1 & -7 & 2 & 1.1 & 1547 \\
\hline 64823915710 & 3.7 & +3 & 3 & 0.4 & 1765 \\
\hline 94375210168 & 4.1 & -1 & 4 & - & 1788 \\
\hline 87413652109 & 2.5 & -3 & 2 & 1.1 & 1678 \\
\hline 25791810364 & 3.6 & +3 & 3 & 0.4 & 1686 \\
\hline 61397105284 & 3.6 & -1 & 3 & 0.4 & 1654 \\
\hline 59268317104 & 3.9 & +1 & 4 & - & 1545 \\
\hline \multicolumn{7}{|c}{$C_{E}$} \\
\hline
\end{tabular}

Within the population indexed by the deviation, solutions with similar deviation are clustered together, and each cluster is separated by at least a single $C_{A}$ as seen in (5).

$$
\begin{aligned}
& \left(\delta_{1}, \delta_{2}, \ldots, \delta_{i}\right) \stackrel{C_{A}}{\leftrightarrow}\left(\delta_{i+1}, \delta_{i+2}, \ldots, \delta_{2 i}\right) \stackrel{C_{A}}{\leftrightarrow} \\
& \ldots \stackrel{C_{A}}{\leftrightarrow}\left(\delta_{3 i+1}, \delta_{3 i 2}, \ldots, \delta_{4 i}\right)
\end{aligned}
$$

An illustrative example is presented to showcase how this approach works. Assume a group of solutions as presented in Table 3.

Each solution is evaluated for its deviation and spread as given in columns 2 and 3. Column 4 gives the cluster to which the solution belongs, and column 5 gives the value of $C_{A}$ by which each cluster is separated from each other. The graphical representation is given in Fig. 1.

The second controlling factor is the Cluster Edge $C_{E}$. Whereas $C_{A}$ is the mapping of individual solutions, $C_{E}$ is the measure of the entire population. $C_{E}$ is the measure of the deviation of the fitness of the population and to prevent the population from stagnating to any fitness minima.

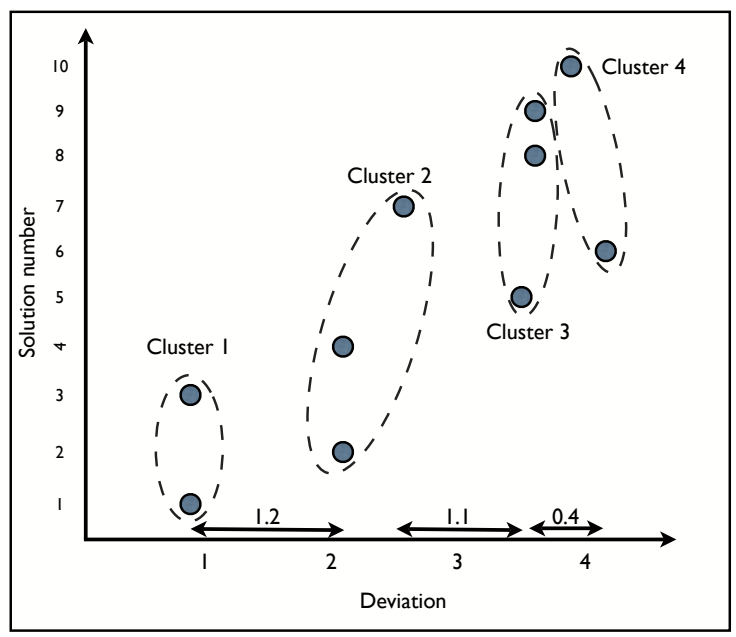

Figure 1: Graphical representation of population
Table 4: Selection criteria

\begin{tabular}{|c|c|}
\hline Variables & Criteria \\
\hline Fitness & Improves clusters best solution \\
\hline$C_{A}$ & Increases the value of $C_{A}$ \\
\hline$C_{E}$ & Decreases \\
\hline
\end{tabular}

Table 5: Deletion criteria

\begin{tabular}{|c|c|}
\hline Variables & Criteria \\
\hline Life & High \\
\hline Offspring & Low \\
\hline$C_{A}$ & Decreases \\
\hline
\end{tabular}

\subsection{Selection and Deletion}

Selection of the next generation is based on a tier-based system. If the new solution improves on the global minima, it is then accepted in the solution. Otherwise, competing clusters jockey for the new solution. Initially the solution is mapped for its deviation. This deviation is then mapped to the corresponding cluster.

Within the cluster, the placement of the solution is evaluated. If the new solution corresponds to an existing solution, or reduces the threshold $C_{A}$ value of the cluster, then it is discarded.

The solution is accepted if it improves on the $C_{A}$ value of the cluster (hence improving diversity) and also to some extent keeps the balance of the $C_{E}$. If the cluster has less than average solutions, then the new solution is admitted.

Table 4 gives the selection criteria. Once the solution is added to the cluster, another solution can be discarded. This solution is usually elected from the middle placed solutions in the cluster, whose fitness is not in the top 5\% of the population. If no such solutions exist, then the average rated solution is removed. Solution with high Life and low Offspring are discarded, since they are considered dormant within the cluster.

Table 5 gives the deletion criteria.

\section{DIFFERENTIAL EVOLUTION}

Differential Evolution (DE) given by Price (1999), is the heuristic which was used in conjunction with the clustered population. DE uses a vector perturbation methodology for crossover.

Each solution is visualized as a vector in search space. A new vector is created by the combination of four unique vectors. A schematic of DE applied to clustering is given in Fig. 2.

There are ten working strategies for $\mathrm{DE}$, but the one selected for implementation is the $\mathrm{DE} / \mathrm{rand} / 2 /$ bin represented as in (6):

$U_{i, G+1}=x_{\text {best }, G}+F \cdot\left(x_{j, r_{1}, G}-x_{j, r_{2}, G}-x_{j, r_{3}, G}-x_{j, r_{4}, G}\right)$

This strategy was selected since it maps to the four unique clusters in the $S P$. The best solution is selected from the entire $S P$ based on fitness value. Then, each random solution is selected from each distinct cluster. Again 


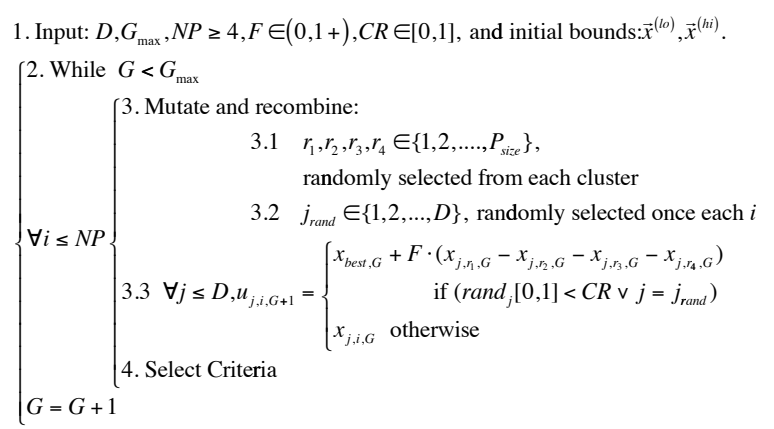

Figure 2: DE selection

the selected values are checked for opposing spread. If the spread is identical, then a second round of selection occurs. A schematic is given in Fig. 3. The selection

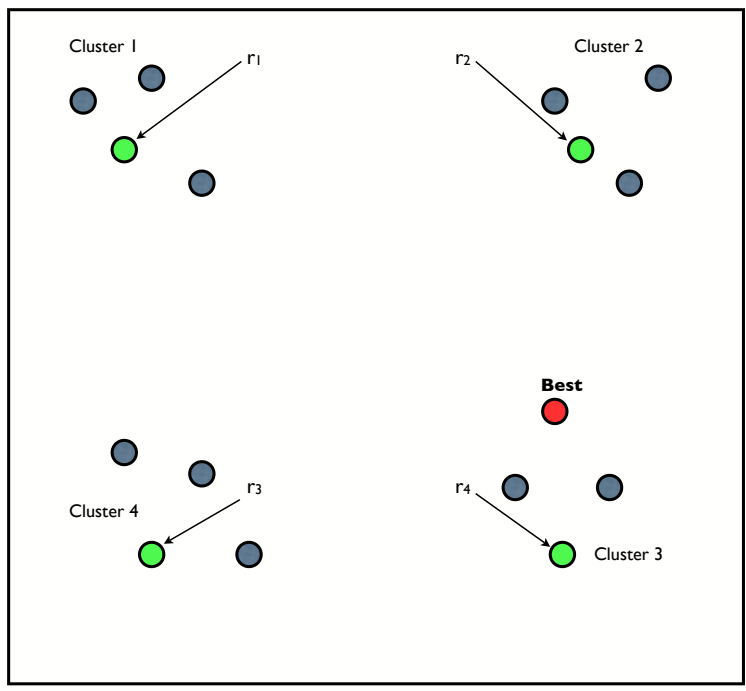

Deviation solution space

Figure 3: DE selection

of the cluster is random, so $r_{1}$ can be selected from any cluster with no preference. These values are subtracted given as $x_{j, r_{1}, G}-x_{j, r_{2}, G}-x_{j, r_{3}, G}-x_{j, r_{4}, G}$. The resulting value is multiplied by the scaling factor $F$ and added to the best solution as given in Fig. 4. The resulting value is only accepted in the new solution if a generated random number is below the given threshold provided by the controlling parameter of $C R$. This procedure provides added stochasticity to the heuristic.

As can be envisioned, the resulting value obtained is a real number. The repairment routine of Onwubolu and Davendra (2009) is used for repairment of the solutions.

Since it is also possible to obtain identical values in the solution after DE crossover, the repairment routine is applied to repair the solutions.

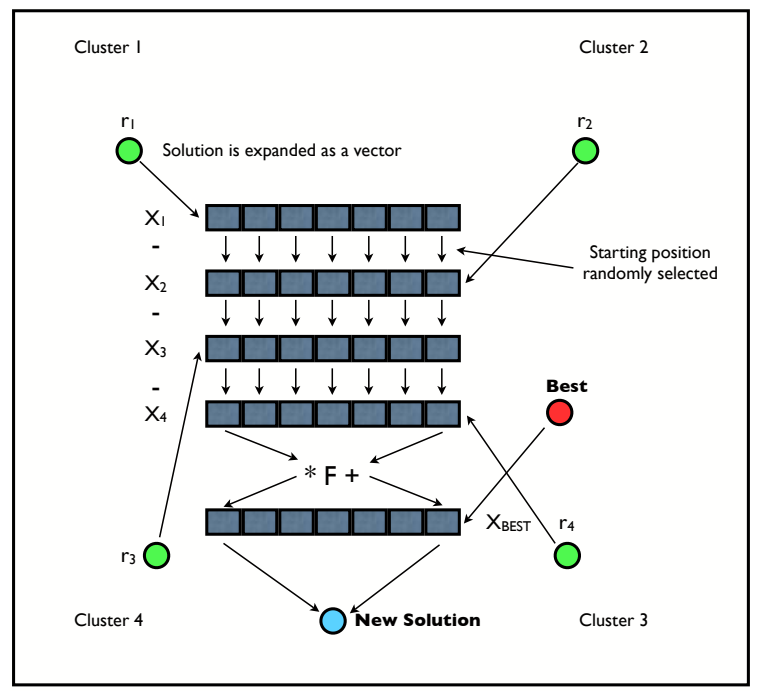

Deviation solution space

Figure 4: DE crossover

\section{GENERAL TEMPLATE}

Collating all the piecewise explanation, a general generic template is now described.

1. Initialize: Assign the problem size $n$, population size $P_{\text {size }}$, sub population sizes $S P_{\text {struct }} S P_{\text {rand }}$, and the control parameters of $C_{A}$ and $C_{E}$.

2. Generate: Randomly create $S P_{\text {rand }}$, halves the size of $P_{\text {size }}$, and then structurally create $S P_{\text {struct }}$. These two form the basis of the population.

3. Calculate: Calculate the deviation and spread of each solution. Taking deviation values, configure the population into four clusters. The minimal separation value between the clusters is assigned as $C_{A}$. Taking the entire $S P$, the standard deviation of the fitness is computed. This is labelled as the $C_{E}$.

\section{Generation}

(a) DE is applied to each $S P$ in turn.

(b) The new solution is calculated for its deviation and spread.

(c) Using the selection criteria, the solution is placed within the cluster corresponding to its deviation. If replicated solutions exist, then it is discarded. Selection is based on fitness and the move of the $C_{A}$ and $C_{E}$.

5. Re-calculation: The SP is re-calculated for its cluster boundaries.

6. Dynamic clustering: If the value of $C_{A}$ has decreased, then the boundary solutions are reconfigured. The $C_{E}$ value is calculated for the new population. 


\section{FLOW SHOP SCHEDULING}

One of the common manufacturing tasks is scheduling. Often in most manufacturing systems, a number of tasks have to be completed on every job. Usually all these jobs have to follow the same route through the different $\mathrm{ma}$ chines, which are set up in a series. Such an environment is called a flow shop (FSS) Pinedo (1995).

The standard three-field notation Lawler et al. (2006) used is that for representing a scheduling problem as $\alpha|\beta| F(C)$, where $\alpha$ describes the machine environment, $\beta$ describes the deviations from standard scheduling assumptions, and $F(C)$ describes the objective $C$ being optimised. This research solves the generic flow shop problem represented as $n / m / f \| F\left(C_{\max }\right)$.

Stating these problem descriptions more elaborately, the minimization of completion time (makespan) for a flow shop schedule is equivalent to minimizing the objective function $\Im$ :

$$
\begin{gathered}
\Im=\sum_{j=1}^{n} C_{m, j} \\
\text { s.t. } \quad C_{i, j}=\max \left(C_{i-1, j}, C_{i, j-1}\right)+P_{i, j}
\end{gathered}
$$

where $C_{m, j}=$ is the completion time of job j, $C_{1,1}=k$ (any given value), $C_{i, j}=\sum_{k=1}^{j} C_{1, k} ; C_{j, i}=\sum_{k=1}^{i} C_{k, 1}$, $i \Rightarrow$ machine number, $j \Rightarrow$ job in sequence, $P_{i, j} \Rightarrow$ processing time of job $j$ on machine $i$. For a given sequence, the mean flow time, $M F T=\frac{1}{n} \sum_{i=1}^{m} \sum_{j=1}^{n} c_{i, j}$, while the condition for tardiness is $c_{m, j}>d_{j}$. The constraint of Equation 8 applies to these two problem descriptions.

\section{EXPERIMENTATION}

The first sets of Flowshop scheduling benchmark problems are Car Carlier (1978), Rec Reeves (1995) and Hel Heller (1960) benchmark sets. A total of 31 instances exist, each of varying size and difficulty Ponnambalam et al. (2001).

Comparison is done with other published heuristics on the same problem instances. Comparison of the $D E_{C l u s}$ heuristics is done with the Improved Genetic Algorithm (IGA) and Multiagent Evolutionary Algorithm (MAEA) $\mathrm{Hu}$ et al. (2006) and the Hybrid Genetic Algorithm (HGA) and Othogonal Genetic Algorithm (OGA) of Tseng and Lin (2006). The results are given in Table 6.

The results of DE were obtained from Davendra and Onwubolu (2007). In 11 instances, the optimal value was obtained, and on average the percentage increase was below $1 \%$. Using clustering, $D E_{\text {clus }}$ markedly improves all the solutions. This is clearly seen in the large problems sizes of 50 jobs and more. The improvement is clearly in excess of $1.5 \%$.
Table 7: Comparison of Taillard Instances

\begin{tabular}{|l|l|l|l|l|l|l|l|l|}
\hline & \multicolumn{2}{|c|}{$P S O_{s p v}$} & \multicolumn{2}{c|}{$D E_{\text {spv }+e x}$} & \multicolumn{2}{c|}{$D E$} & \multicolumn{2}{c|}{$D E_{\text {clus }}$} \\
\hline Problem & $\Delta$ avg & $\Delta$ std & $\Delta$ avg & $\Delta$ std & $\Delta$ avg & $\Delta$ std & $\Delta$ avg & $\Delta$ std \\
\hline 20x5 & 1.71 & 1.25 & 0.69 & 0.64 & 0.98 & 0.66 & $\mathbf{0 . 5 5}$ & 0.71 \\
\hline 20x10 & 3.28 & 1.19 & 2.01 & 0.93 & 1.81 & 0.77 & $\mathbf{1 . 3 2}$ & 0.98 \\
\hline 20x20 & 2.84 & 1.15 & 1.85 & 0.87 & 1.75 & 0.57 & $\mathbf{0 . 9 8}$ & 1.32 \\
\hline 50x5 & 1.15 & 0.7 & 0.41 & 0.37 & 0.4 & 0.36 & $\mathbf{0 . 3 3}$ & 0.76 \\
\hline 50x10 & 4.83 & 1.16 & $\mathbf{2 . 4 1}$ & 0.9 & 3.18 & 0.94 & 3.13 & 0.77 \\
\hline 50x20 & 6.68 & 1.35 & $\mathbf{3 . 5 9}$ & 0.78 & 4.05 & 0.65 & 3.67 & 0.56 \\
\hline 100x5 & 0.59 & 0.34 & $\mathbf{0 . 2 1}$ & 0.21 & 0.41 & 0.29 & 0.38 & 0.54 \\
\hline 100x10 & 3.26 & 1.04 & 1.41 & 0.57 & 1.46 & 0.36 & $\mathbf{1 . 3 1}$ & 0.32 \\
\hline 100x20 & 7.19 & 0.99 & 3.11 & 0.55 & 3.61 & 0.36 & $\mathbf{2 . 2 3}$ & 0.45 \\
\hline 200x10 & 2.47 & 0.71 & 1.06 & 0.35 & 0.95 & 0.18 & $\mathbf{0 . 6 9}$ & 0.54 \\
\hline
\end{tabular}

In general comparison with published results, the clustered approach of $D E_{\text {clus }}$ is one of the top two performing heuristics. MAEA approach is the best comparative heuristic and in comparison with $D E_{c l u s}$, even though MAEA obtains more optimal solutions, $D E_{c l u s}$, performs more consistently in large problems.

The second set of benchmark problems is referenced from Taillard (1993). These sets of problems have been extensively evaluated by Reeves and Yamada (1998). This benchmark set contains 120 particularly hard instances each of 10 different size, selected from a large number of randomly generated problems.

The results are tabulated as quality solutions with the percentage relative increase in makespan with respect to the upper bound provided by Taillard (1993). To be specific the formulation is given as:

$$
\Delta_{a v g}=\frac{(H-U) \times 100}{U}
$$

where $H$ denotes the value of the makespan that is produced by the utilized algorithm and $U$ is the upper bound or the lower bound as computed.

Comparison is done with the results obtained for $D E_{\text {clus }}$ with those produced by Particle Swarm Optimization $P S O_{s p v}$ and DE with local search $D E_{\text {spv+exchange }}$ as in Tasgetiren et al. (2004b) and Tasgetiren et al. (2004a) and compared in Table 7.

$D E_{\text {clus }}$ obtains better results in the instances of

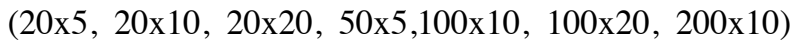
and $D E_{s p v+e x c h a n g e}$ performing best in the remaining $(50 \times 10,50 \times 20,100 \times 5)$ instances. The advantage of $D E_{\text {spv+exchange }}$ is the fact that it employs local search, whereas $D E_{\text {clus }}$ is employing 2-opt local search as given in Onwubolu and Davendra (2009).

DE with clustering produces better results than DE without clustering, validating the approach of embedding population dynamics.

\section{ANALYSIS AND CONCLUSION}

A detailed operational analysis of the heuristic is now presented. A sample program output is presented to illustrate the heuristic behaviour during experimentation. Fig. 5 and Fig. 6 gives the first and final populations mapped on the deviation space. 
Table 6: Comparison of clustered heuristics with other published heuristics

\begin{tabular}{|c|c|c|c|c|c|c|c|c|}
\hline Name & $\mathrm{n} \times \mathrm{m}$ & Cost & H-GA & OGA & IGA & MAEA & DE & DE $E_{\text {Clus }}$ \\
\hline Car1 & $11 \times 5$ & 7038 & $\mathbf{0}$ & $\mathbf{0}$ & $\mathbf{0}$ & $\mathbf{0}$ & $\mathbf{0}$ & $\mathbf{0}$ \\
\hline Car2 & $13 \times 4$ & 7166 & $\mathbf{0}$ & $\mathbf{0}$ & $\mathbf{0}$ & $\mathbf{0}$ & $\mathbf{0}$ & $\mathbf{0}$ \\
\hline Car3 & $12 \times 5$ & 7312 & $\mathbf{0}$ & $\mathbf{0}$ & $\mathbf{0}$ & $\mathbf{0}$ & $\mathbf{0}$ & $\mathbf{0}$ \\
\hline Car4 & $14 \times 4$ & 8003 & $\mathbf{0}$ & $\mathbf{0}$ & $\mathbf{0}$ & $\mathbf{0}$ & $\mathbf{0}$ & $\mathbf{0}$ \\
\hline Car5 & $10 \times 6$ & 7720 & $\mathbf{0}$ & $\mathbf{0}$ & $\mathbf{0}$ & $\mathbf{0}$ & $\mathbf{0}$ & $\mathbf{0}$ \\
\hline Car6 & $8 \times 9$ & 8505 & $\mathbf{0}$ & $\mathbf{0}$ & $\mathbf{0}$ & $\mathbf{0}$ & $\mathbf{0}$ & $\mathbf{0}$ \\
\hline Car7 & $7 \times 7$ & 6590 & $\mathbf{0}$ & $\mathbf{0}$ & $\mathbf{0}$ & $\mathbf{0}$ & $\mathbf{0}$ & $\mathbf{0}$ \\
\hline Car8 & $8 \times 8$ & 8366 & $\mathbf{0}$ & $\mathbf{0}$ & $\mathbf{0}$ & $\mathbf{0}$ & $\mathbf{0}$ & $\mathbf{0}$ \\
\hline Rec01 & $20 \times 5$ & 1247 & 0 & 0.04 & $\mathbf{0}$ & $\mathbf{0}$ & $\mathbf{0}$ & $\mathbf{0}$ \\
\hline Rec03 & $20 \times 5$ & 1109 & $\mathbf{0}$ & $\mathbf{0}$ & $\mathbf{0}$ & $\mathbf{0}$ & $\mathbf{0}$ & $\mathbf{0}$ \\
\hline Rec05 & $20 \times 5$ & 1242 & 0.08 & 0.21 & $\mathbf{0}$ & $\mathbf{0}$ & $\mathbf{0}$ & $\mathbf{0}$ \\
\hline Rec07 & $20 \times 10$ & 1566 & $\mathbf{0}$ & 0.79 & $\mathbf{0}$ & $\mathbf{0}$ & $\mathbf{0}$ & $\mathbf{0}$ \\
\hline Rec09 & $20 \times 10$ & 1537 & $\mathbf{0}$ & 0.35 & $\mathbf{0}$ & $\mathbf{0}$ & $\mathbf{0}$ & $\mathbf{0}$ \\
\hline Rec11 & $20 \times 10$ & 1431 & $\mathbf{0}$ & 0.91 & $\mathbf{0}$ & $\mathbf{0}$ & $\mathbf{0}$ & $\mathbf{0}$ \\
\hline Rec13 & $20 \times 15$ & 1930 & 0.52 & 1.08 & 0.62 & $\mathbf{0}$ & 0.45 & 0.31 \\
\hline Rec15 & $20 \times 15$ & 1950 & 0.92 & 1.23 & 0.46 & $\mathbf{0}$ & 0.32 & 0.28 \\
\hline Rec17 & $20 \times 15$ & 1902 & 1.26 & 2.08 & 1.73 & $\mathbf{0}$ & 0.29 & 0.28 \\
\hline Rec19 & $30 \times 10$ & 2093 & 0.38 & 1.76 & 1.09 & $\mathbf{0 . 2 8}$ & 0.42 & 0.338 \\
\hline Rec21 & $30 \times 10$ & 2017 & 0.89 & 1.64 & 1.44 & 0.44 & 0.39 & $\mathbf{0 . 3 8}$ \\
\hline Rec23 & $30 \times 10$ & 2011 & 0.45 & 1.9 & 0.45 & 0.44 & $\mathbf{0 . 2 1}$ & $\mathbf{0 . 2 1}$ \\
\hline Rec25 & $30 \times 15$ & 2513 & 1.03 & 2.67 & 1.63 & 0.43 & 0.32 & $\mathbf{0 . 2 9}$ \\
\hline Rec27 & $30 \times 15$ & 2373 & 1.18 & 2.09 & 0.8 & 0.56 & 0.42 & $\mathbf{0 . 2 7}$ \\
\hline Rec29 & $30 \times 15$ & 2287 & 1.05 & 3.28 & 1.53 & 0.78 & 0.61 & $\mathbf{0 . 3 4}$ \\
\hline Rec31 & $50 \times 10$ & 3045 & 0.56 & 1.49 & 0.49 & $\mathbf{0 . 1}$ & 0.7 & 0.32 \\
\hline Rec33 & $50 \times 10$ & 3114 & $\mathbf{0}$ & 1.87 & 0.13 & $\mathbf{0}$ & 0.84 & 0.28 \\
\hline Rec35 & $50 \times 10$ & 3277 & $\mathbf{0}$ & $\mathbf{0}$ & $\mathbf{0}$ & $\mathbf{0}$ & 0.91 & 0.27 \\
\hline Rec37 & $75 \times 20$ & 4951 & 2.54 & 3.41 & 2.26 & 2.72 & 1.32 & $\mathbf{0 . 3 3}$ \\
\hline Rec39 & $75 \times 20$ & 5087 & 1.79 & 2.28 & 1.14 & 1.61 & 1.56 & $\mathbf{0 . 2 9}$ \\
\hline Rec41 & $75 \times 20$ & 4960 & 2.82 & 3.43 & 3.27 & 2.7 & 1.98 & $\mathbf{0 . 2 8}$ \\
\hline He101 & $100 \times 10$ & 513 & - & - & - & $\mathbf{0 . 3 8}$ & 2.1 & 0.53 \\
\hline He102 & $20 \times 10$ & 135 & - & - & - & $\mathbf{0}$ & 1.97 & $\mathbf{0}$ \\
\hline
\end{tabular}

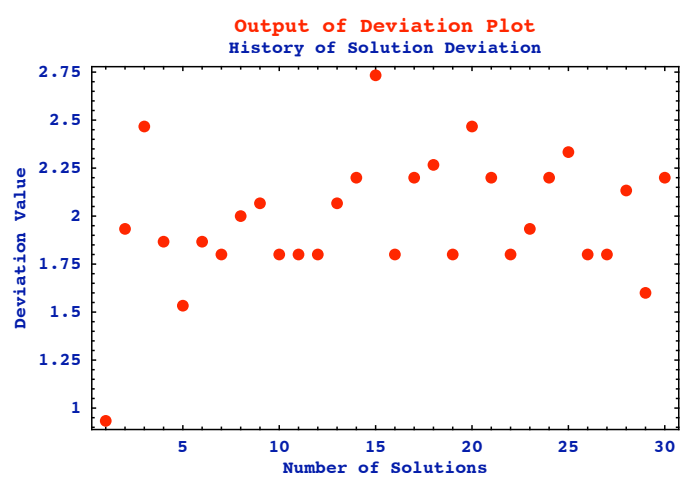

Figure 5: $D E_{\text {clust }}$ initial population

The evident feature of the two plots is that the diversity of the solutions increased over the generations. Another feature is that separate search spaces were explored since the deviation value increased over the generations.

The second controlling parameter of $C_{E}$, which controls the grouping of the population, is given in Fig. 7. As seen from 7, there is a general decline of $C_{E}$ within $D E_{\text {clust }}$. This is evident for a major migration of the cluster.

The history of the best solution is presented in Fig. 8 . Clustering improves solution diversity, however the importance of $C_{E}$ can be easily seen when comparing the two plots of $C_{E}$ in Fig. 7 and best solution in Fig. 8. A

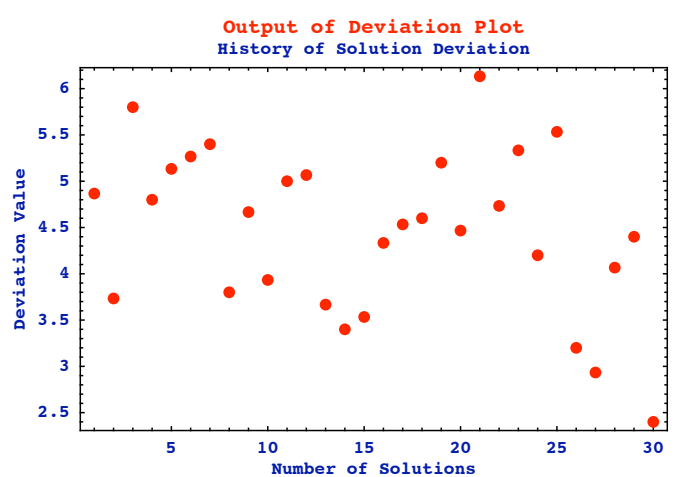

Figure 6: $D E_{\text {clust }}$ final population

correlation is observed between the two plots, since any major activity in $C_{E}$ has a flow on impact to the best individual. The explanation is that once the cluster drifts into new solution spaces, a rapid divergence occurs, which leads to possibly discovery of new optimal solutions.

Once the local space is searched, a different directional search occurs, which generally results in an increase of the value of $C_{E}$, as observed from the two different plots.

Another unique feature of the experimentation as the fact that approximately $45 \%$ of solutions were obtained from the structured population. This validates the approach of utilising a structured initial population alongside a random population. 


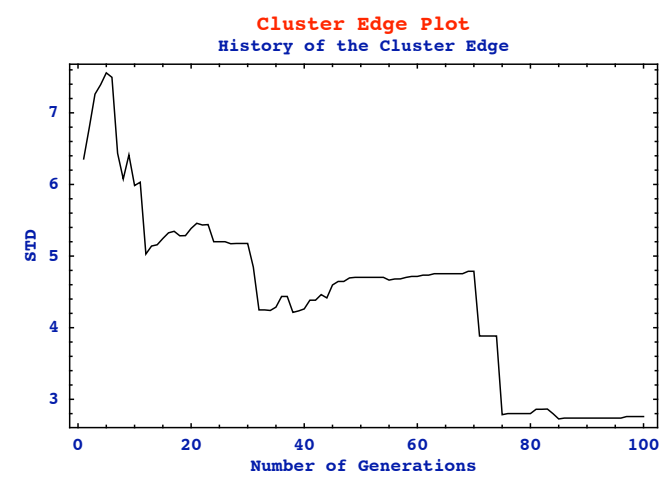

Figure 7: $D E_{\text {clust }} C_{E}$ values over the generations

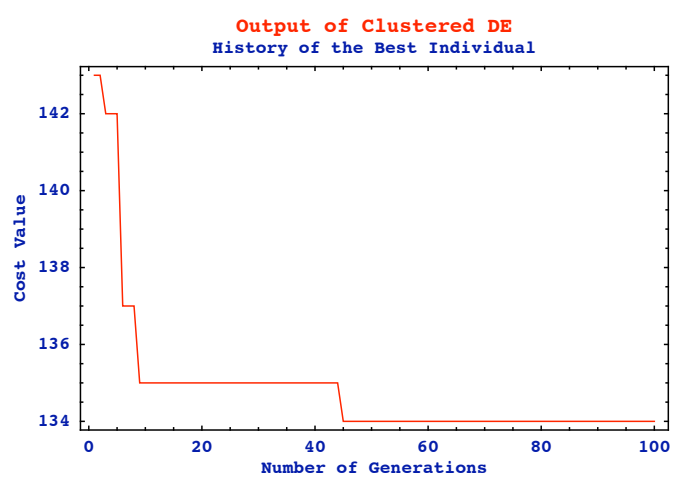

Figure 8: Best solution history of $D E_{\text {clust }}$

\section{REFERENCES}

Carlier, J. (1978). Ordonnancements a contraintes disjonctives. Operations Research, 12:333-351.

Davendra, D. and Onwubolu, G. (2007). Flow shop scheduling using enhanced differential evolution. In Proc.21 European Conference on Modeling and Simulation, pages 259264, Prague, Czech Rep.

Dreo, J., Petrowski, A., Siarry, P., and Taillard, E. (2006). Metaheuristics for Hard Optimization. Springer-Verlag, Germany.

Heller, J. (1960). Some numerical experiments for an mj flow shop and its decision- theoretical aspects. Operations Research, 8:178-184.

Hochbam, D., editor (1997). Approximation Algorithms for NP Hard Problems. PWS Publishing Company., Washington, USA.

Hu, K., Li, J., Liu, J., and Jiao, L. (2006). Permutation flow-shop scheduling based on multiagent evolutionary algorithm. In Sattar, A. and Kang, B., editors, AI 2006: Advances in Artificial Intelligence. Springer-Verlag, Berlin, Germany.

Lawler, E. et al. (2006). Sequencing and scheduling: algorithms and complexity. In Graves, S., Kan, A. R., and Zipkin, P., editors, In Logistics of Production and Inventory. North Holland, Amsterdam, Netherlands.
Onwubolu, G. (2002). Emerging Optimization Techniques in Production Planning and Control. Imperial Collage Press, London, England.

Onwubolu, G. and Davendra, D., editors (2009). Differential Evolution: A Handbook for Global Permutation-based Combinatorial Optimization. Springer, Germany.

Pinedo, M. (1995). Scheduling: theory, algorithms and systems. Prentice Hall, New Jersey.

Ponnambalam, S., Aravindan, P., and Chandrasekhar, S. (2001). Constructive and improvement flow shop scheduling heuristic: an extensive evaluation. Production Planning and Control, 12:335-344.

Price, K. (1999). An introduction to differential evolution. In Corne, D., Dorigo, M., and Glover, F., editors, New ideas in Optimisation. McGraw Hill, UK.

Reeves, C. (1995). A genetic algorithm for flowshop sequencing. Computers and Operations Research, 22:5-13.

Reeves, C. and Yamada, T. (1998). Genetic algorithms, path relinking and flowshop sequencing problem. Evolutionary Computation, 6:45-60.

Taillard, E. (1993). Benchmarks for basic scheduling problems. European Journal of Operations Research, 64:278-285.

Tasgetiren, M., Liang, Y.-C., Sevkli, M., and Gencyilmaz, G. (2004a). Particle swamp optimization algorithm for permutative flowshops sequencing problems. In Differential Evolution Algorithm for Permutative Flowshops Sequencing Problem with Makespan Criterion, pages 442-452, Sakaraya, Turkey.

Tasgetiren, M., Sevkli, M., Liang, Y.-C., and Gencyilmaz, G. (2004b). Particle swamp optimization algorithm for permutative flowshops sequencing problems. In 4th International Workshops on Ant Algorithms and Swamp Intelligence, pages 389-390, Brussel, Belgium.

Tseng, L. and Lin, T. (2006). A hybrid genetic algorithm for the flow-shop scheduling problem. Lecture Notes in Computer Science, pages 218-227.

\section{AUTHOR BIOGRAPHIES}

DONALD DAVENDRA has a BSc, PGD and MSc in Computing Science from the University of the South Pacific. He is currently completing his $\mathrm{PhD}$ in Technical Cybernatics at Tomas Bata University. His email is davendra@fai.utb.cz.

IVAN ZELINKA is Assoc. Professor of Informatics and Head of Department of Applied Informatics at Tomas Bata University. His email is zelinka@fai.utb.cz.

GODFREY ONWUBOLU is a Professor of Manufacturing and Director of Knowledge Managament and Mining division of DaySpring Global Multinational Inc. His email is onwubolu_g@dsgm.ca. 